\title{
Implantação da sistematização da assistência de enfermagem: opinião de uma equipe de enfermagem hospitalar
}

Recebido em: 23/04/2012

Aceito em: 12/07/2012

\author{
Suzana de Oliveira Mangueira ${ }^{1}$ \\ Juliana Tallyta Silva de Lima² \\ Soraia Lins de Arruda Costa ${ }^{3}$ \\ Maria Miriam Lima da Nóbrega ${ }^{4}$ \\ Marcos Venícios de Oliveira Lopes 5
}

O estudo objetiva analisar a opinião da equipe de enfermagem acerca do processo de implantação da Sistematização da Assistência de Enfermagem em uma instituição hospitalar. Trata-se de um estudo descritivo exploratório, qualitativo. Os resultados apontaram que os profissionais reconhecem a importância da Sistematização da Assistência de Enfermagem como instrumento para eficiência e eficácia das ações, organização e padronização da assistência, individualização e continuidade do cuidado. É esperado que a sua implantação traga benefícios para pacientes, profissionais e instituição. As dificuldades referem-se ao déficit de recursos materiais e humanos. Espera-se que estes dados possam subsidiar ações que minimizem estes obstáculos.

Descritores: Enfermagem, Equipe de Enfermagem, Processos de Enfermagem.

\section{Implementation of nursing care systematization: a view hospital nursing team}

The study aims to analyze the opinion of the nursing team about the process of implementation of the nursing care systematization in a hospital. This is a qualitative descriptive exploratory study. The results indicated that the team recognizes the importance of the nursing care systematization as a tool for efficiency and effectiveness of actions, organization and standardization of care, individualization and continuity of care. It's expected that its implementation will bring benefits to patient, professionals and institution. The difficulties relate to lack of material and human resources. It's hoped that these data may support actions to minimize these obstacles.

Descriptors: Nursing, Nursing Team, Nursing Process.

\section{Aplicación de la sistematización de la asistencia de enfermería: opinión del equipo de enfermería de un hospital}

El estudio tiene como objetivo analizar la opinión del equipo de enfermería sobre el proceso de implementación de la sistematización de la asistencia de enfermería en un hospital. Este es un estudio descriptivo exploratorio cualitativo. Los resultados indicaron que el personal reconozca la importancia de la sistematización de la asistencia de enfermería como una herramienta para la eficiencia y la eficacia de las acciones, la organización y estandarización de los cuidados, la individualización y la continuidad de la atención. Se espera que su aplicación traiga beneficios para pacientes, profesionales e institución. Las dificultades se relacionan con la falta de recursos materiales y humanos. Se espera que estos datos podrían apoyar las acciones para minimizar estos. Descriptores: Enfermería, Grupo de Enfermería, Procesos de Enfermería.

\section{INTRODUÇÃO}

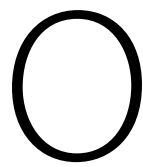
processo de enfermagem é uma forma sistemática e dinâmica de prestação de cuidados de enfermagem, composto por cinco etapas inter-relacionadas: investigação, diagnóstico de enfermagem, planejamento, implementação e avaliação(1). As primeiras tentativas de sistematizar os cuidados de enfermagem no Brasil foram propostas por Horta, na década de $1970^{(2)}$, porém, só em 1986, com a Lei que regulamenta o Exercício Profissional da Enfermagem, n. ${ }^{7}$ 7498/86, a consulta e a prescrição de enfermagem foram destacadas como atividades privativas do enfermeiro $^{(3)}$. Desde então, as discussões sobre a temática têm sido debatidas e tornam cada vez mais clara a necessidade de uma assistência de enfermagem menos intuitiva e mais científica.

Em 2002, o Conselho Federal de Enfermagem (Cofen) estabelece a obrigatoriedade de implantação da Sistematização da Assistência de Enfermagem (SAE) em todas as instituiçōes de saúde no Brasil, por meio da Resolução n. $272 / 2002$. Em 2009, o Cofen a reformula e amplia a obrigatoriedade da SAE e a implementação do Processo de Enfermagem para todos os ambientes, públicos ou privados, em que ocorre o cuidado

1 Enfermeira. Mestre em Enfermagem pela UFPB. Doutoranda em Enfermagem pela UFC. Professora Assistente do Núcleo de Enfermagem do Centro Acadêmico de Vitória/ UFPE. E-mail: suzanaom@hotmail.com

2 Enfermeira assistencial do Hospital da Polícia Militar, Recife-PE.

3 Enfermeira. Mestranda do Programa de Pós-graduação em Saúde Humana e Meio Ambiente do Centro Acadêmico de Vitória/UFPE.

4 Enfermeira. Doutora em Enfermagem pela UNIFESP. Professora Associada do Departamento de Enfermagem da UFPB.

5 Enfermeiro. Doutor em Enfermagem pela UFC. Professor Associado do Departamento de Enfermagem da UFC. 
profissional de Enfermagem, por meio da Resolução n. ${ }^{\circ}$ $358 / 2009^{(4)}$. No intuito de atender a essas exigências, instituições de saúde têm buscado estratégias para implantar a SAE e têm enfrentado diversos obstáculos.

No estado de Pernambuco, destaca-se a experiência de um hospital geral público estadual, que iniciou as atividades para implantação da $\mathrm{SAE}$, como preconizado pelo Cofen. No entanto, ainda são escassos os subsídios para a sua efetivação, aliados aos entraves inerentes à própria instituição. $O$ processo de implantação da SAE é bastante complexo, o que torna fundamental conhecer a estrutura institucional e os aspectos que possam contribuir para facilitar ou prejudicar a sua implantação(5).

Assim, o estudo objetiva analisar a opinião da equipe de enfermagem sobre a da SAE no referido hospital, com vistas a compreender os benefícios gerados por ela, as expectativas e motivações de profissionais, bem como identificar as dificuldades encontradas nesta construção.

\section{MÉTODO}

Trata-se de um estudo descritivo exploratório com abordagem qualitativa. Buscou mostrar as características do fenômeno estudado a partir da análise de discursos e compreender sua importância e não a frequência de sua ocorrência ${ }^{(6)}$.

O estudo foi desenvolvido em um hospital geral estadual, localizado no estado de Pernambuco, que presta assistência nas diversas especialidades. A equipe de Enfermagem é composta por 36 enfermeiros e 72 técnicos de enfermagem. 0 número de participantes foi delimitado por acessibilidade e considerou o critério de saturação das respostas. Participaram oito profissionais, destes, cinco enfermeiros (E1, E2, E3, E4 e E5) e três técnicos de enfermagem (TE1, TE2 e TE3) que atuam no setor de clínica médica, por ser a unidade pioneira de implementação da SAE hospital.

Para a coleta de dados, foi utilizado um questionário que continha questões referentes à caracterização dos participantes e questões relativas à opinião dos profissionais sobre a implantação da SAE, que contemplou sua importância, os fatores que motivam essa implantação na instituição e as dificuldades encontradas. $\mathrm{O}$ instrumento foi submetido a um pré-teste com cinco profissionais de enfermagem do hospital e, após ajustes, foram distribuídos 30 questionários, com prazo estipulado para a sua devolução preenchido. Apenas 11 foram devolvidos e, destes, três foram excluídos por apresentar questões em branco, o que resultou em oito questionários para análise.

A coleta de dados realizou-se após a aprovação do projeto pelo Comitê de Ética em Pesquisa do Hospital Otávio de Freitas, em Recife-PE, sob protocolo n. ${ }^{\circ}$ 0.18.08.2010. Os participantes foram esclarecidos quanto ao estudo e a seus direitos, conforme a Resolução 196/96 do Conselho Nacional de Saúde (7) e assinaram o Termo de Consentimento Livre e Esclarecido.

Os dados coletados foram analisados qualitativamente, com base na técnica de análise temática de conteúdo, que se trata de uma técnica de análise das comunicações. Essa técnica perpassa por três polos cronológicos: a pré-análise, a exploração do material e o tratamento dos resultados, a inferência e a interpretação( ${ }^{(8)}$.

A pré-análise consistiu em uma leitura flutuante dos questionários e observou-se a relação entre o seu conteúdo e os objetivos propostos. Foram destacados os fragmentos mais relevantes que constituíram corpus de análise. Durante o segundo polo cronológico, a exploração do material, os fragmentos do corpus foram agrupados, por similaridade, em subcategorias e estas, em três categorias mais abrangentes. O tratamento dos resultados, a inferência e a interpretação basearam-se na apresentação dos resultados em quadros e foram feitas inferências específicas sobre o conteúdo. Buscou-se contemplar o objetivo do estudo a partir da interpretação e discussão com base na literatura.

\section{RESULTADOS}

Os participantes eram, em sua maioria, do sexo feminino, na faixa etária predominante de 31-40 anos de idade, com média de cinco anos de formação e experiência na função e 2,5 anos

Categoria 1: Importância da SAE para a prática da Enfermagem

\begin{tabular}{|c|c|}
\hline Subcategorias & Unidades Temáticas \\
\hline $\begin{array}{l}\text { Eficiência e } \\
\text { eficácia da } \\
\text { assistência de } \\
\text { enfermagem }\end{array}$ & $\begin{array}{l}\text { A assistência ficará mais completa, } \\
\text { sistemática e complementar [...]. (E2) } \\
\text { [...] beneficiando a recuperação dos } \\
\text { pacientes de uma maneira rápida, } \\
\text { eficiente e eficaz. (TE1) } \\
\text { Facilita tanto para o enfermeiro quanto } \\
\text { para o pessoal técnico realizar os } \\
\text { procedimentos. (E5) } \\
\text { É uma maneira de contribuir com uma } \\
\text { recuperação mais rápida e eficaz dos } \\
\text { pacientes [...]. (E1) }\end{array}$ \\
\hline $\begin{array}{l}\text { Organização e } \\
\text { padronização da } \\
\text { assistência }\end{array}$ & $\begin{array}{l}\text { [...] realizando o diagnóstico e } \\
\text { organizando toda assistência de acordo } \\
\text { com os problemas encontrados. (E3) } \\
\text { De forma organizada, seguindo uma só } \\
\text { rotina [...].(TE1) } \\
\text { É uma padronização da assistência e } \\
\text { prescrição da enfermagem. (E5) }\end{array}$ \\
\hline $\begin{array}{l}\text { Individualização } \\
\text { do cuidado }\end{array}$ & $\begin{array}{l}\text { Uma assistência qualificada e } \\
\text { individualizada nas necessidades do } \\
\text { paciente. (TE1) }\end{array}$ \\
\hline $\begin{array}{l}\text { Continuidade da } \\
\text { assistência }\end{array}$ & $\begin{array}{l}\text { [...] de forma que haja continuidade de } \\
\text { plantão a plantão ou de uma equipe para } \\
\text { outra. (E4) }\end{array}$ \\
\hline $\begin{array}{l}\text { Melhoria para a } \\
\text { instituição }\end{array}$ & $\begin{array}{l}\text { [...] melhoria de custo e benefício para a } \\
\text { instituição. (E4) }\end{array}$ \\
\hline
\end{tabular}

Quadro 1 - Distribuição de unidades temáticas por subcategorias referentes à importância da SAE para a prática da Enfermagem

no serviço. Quanto à importância da $\mathrm{SAE}$, todos os profissionais reconheceram os seus benefícios, conforme o Quadro 1. 
Categoria 2: Motivações diante da implantação da SAE na instituição

\begin{tabular}{|c|c|}
\hline Subcategorias & Unidades Temáticas \\
\hline $\begin{array}{l}\text { Melhoria da } \\
\text { assistência de } \\
\text { Enfermagem } \\
\text { para o paciente }\end{array}$ & $\begin{array}{l}\text { [...] otimização da assistência. (E2) } \\
\text { [...] os pacientes haverão de agradecer } \\
\text { tudo e qualquer empenho em } \\
\text { desenvolver meios e recuperação rápida. } \\
\text { (TE1) } \\
\text { [...] a mudança é o melhor planejamento } \\
\text { da assistência. (E3) } \\
\text { Recuperar as doenças já existentes e } \\
\text { aquelas adquiridas no setor. (E1) }\end{array}$ \\
\hline $\begin{array}{l}\text { Melhoria para o } \\
\text { profissional de } \\
\text { Enfermagem }\end{array}$ & $\begin{array}{l}\text { Permite exercer com plenitude meu } \\
\text { papel como enfermeira. (E4) } \\
\text { Vai ser válida a experiência do dever } \\
\text { como norte para as minhas ações. (TE1) }\end{array}$ \\
\hline $\begin{array}{l}\text { Melhoria para a } \\
\text { instituição }\end{array}$ & $\begin{array}{l}\text { Que venha contribuir para uma melhoria } \\
\text { dos setores. (TE1) } \\
\text { [...] quem ganha é o hospital, também. } \\
\text { (TE3) }\end{array}$ \\
\hline
\end{tabular}

Quadro 2 - Distribuição de unidades temáticas por subcategorias referentes às motivações diante da implantação da SAE na instituição

Reconhecer os benefícios da SAE para a prática assistencial reflete nos fatores que motivam os profissionais para a sua implantação no serviço, visto que eles reconhecem que esse processo trará uma melhoria para a assistência prestada ao paciente, os profissionais e a instituição, conforme o Quadro 2.

Categoria 3: Limitaçōes a serem superadas no processo de implantação da SAE na instituição

\begin{tabular}{|l|l|}
\hline $\begin{array}{l}\text { Subcategorias } \\
\text { Déficit de recursos } \\
\text { materiais }\end{array}$ & \begin{tabular}{l}
\multicolumn{1}{c|}{ Unidades Temáticas } \\
Que nós tenhamos recursos materiais \\
{$[. .$.$] (E3)$} \\
Diante das necessidades do serviço, \\
penso que haveria atualmente outras \\
melhorias. (E2) \\
Tem muita coisa que precisa melhorar, \\
aqui é tudo muito precário. (TE2)
\end{tabular} \\
$\begin{array}{l}\text { Déficit de recursos } \\
\text { humanos }\end{array}$ & $\begin{array}{l}\text { Ter enfermeiras em quantidade suficiente } \\
\text { [...] (E5) } \\
\text { Que se reúna uma equipe responsável } \\
\text { pelo serviço, que estude e planeje a } \\
\text { solução mais rápida e adequada [...] (E1) } \\
\text { [...] que seja dinâmico e motivador. (E4) }\end{array}$ \\
\hline
\end{tabular}

Quadro 3 - Distribuição de unidades temáticas por subcategorias referentes às limitações a serem superadas no processo de implantação da SAE na instituição

Apesar de reconhecer os benefícios e estarem motivados para a implementação da $\mathrm{SAE}$, os profissionais sabem quais são as principais limitações para a sua efetivação, apresentadas no Quadro 3.

\section{DISCUSSÃO}

\section{Categoria 1 - Importância da SAE para a prática da Enfermagem}

O reconhecimento dos benefícios gerados pela implementação do processo de enfermagem é importante para a conquista de uma maior autonomia profissional. Sua efetivação evidencia os problemas que a Enfermagem pode identificar e tratar independentemente ${ }^{(9)}$.

O processo de enfermagem é apontado como um instrumento que permite organizar e padronizar a prática profissional. Quando parte de conhecimentos específicos e reflexão crítica e problematizadora do trabalho da Enfermagem, a SAE constitui um instrumento para gerenciamento e otimização da assistência de enfermagem de forma organizada, segura, dinâmica e competente ${ }^{(10)}$. Apesar de haver muitas críticas à forma de sua utilização, o processo de enfermagem tem sido considerado como um provedor da uniformização da linguagem, por sistematizar a prática e ampliar a autonomia profissional ${ }^{(11)}$.

Ao realizar um cuidado sistematizado, o enfermeiro promove a individualização do cuidado e favorece a comunicação entre a equipe. $O$ processo de enfermagem é sustentado por um referencial próprio, que possibilita a união entre teoria e prática. O uso de referenciais teóricos modifica a estrutura da assistência, promove a ação crítica e participativa e exige maior conhecimento da Enfermagem ${ }^{(12)}$.

Quando é implantada e consolidada em uma instituição, a SAE se torna um serviço de qualidade, favorece a diminuição do tempo de internação, a satisfação do cliente, um menor risco de infecção e, consequentemente, a redução de custos $^{(1)}$. Assim, destaca-se que deve haver, além do compromisso dos profissionais de enfermagem, um comprometimento da gestão institucional, de forma a valorizar a SAE e propiciar as condições necessárias para a sua efetivação(13).

\section{Categoria 2 - Motivações diante da implantação da SAE na instituição}

Em relação aos fatores motivadores na perspectiva da implantação da SAE no hospital em estudo, os profissionais esperam que benefícios sejam gerados na sua realidade. Os ganhos para todos os envolvidos são enormes. Quando são analisados aspectos assistenciais e administrativos, fica nítido o aumento da satisfação dos pacientes, a diminuição de iatrogenias, a redução do período de internação dos pacientes, a diminuição das glosas hospitalares, a otimização do trabalho da equipe de enfermagem, a definição do papel do enfermeiro e o aumento da autoestima dos profissionais de enfermagem ${ }^{(14)}$.

Destaca-se que a SAE promove a melhoria na qualidade da assistência, contribui para a autonomia profissional, proporciona aos enfermeiros a flexibilidade do pensamento crítico, melhora a comunicação entre a equipe e previne erros, omissões e repetições desnecessárias. Embora seja mister a importância da $\mathrm{SAE}$, a maioria dos estudos sobre a temática no âmbito nacional retrata as dificuldades de sua implementação, em detrimento aos estudos que traduzem os benefícios gerados por ela em experiências práticas ${ }^{(15)}$. 


\section{Categoria 3 - Limitações a serem superadas no processo de implantação da SAE na instituição}

Os discursos apontam para o déficit de recursos materiais e humanos como os principais entraves para a efetivação da SAE. A carência de material e a estrutura precária induzem os profissionais de enfermagem a afirmar que a SAE não é uma prioridade e ressaltam que deve haver uma melhoria das condições de trabalho para, posteriormente, implementar essa metodologia. A estrutura física é um aspecto pouco citado na literatura, porém, sua análise é importante, com vistas a realizar as adequações necessárias para que cada etapa ocorra de modo satisfatório. Nesse contexto, destaca-se que um dos aspectos primordiais para a viabilidade prática da SAE é o interesse institucional e compromisso da gestão em implantar a proposta, de modo a viabilizar os recursos necessários à sua implementação e manutenção(5).

No entanto, os profissionais de enfermagem têm se esforçado para colocar em prática essa metodologia da assistência, mas têm enfrentado desafios. São apontados como fatores intervenientes na operacionalização do processo de enfermagem: déficit de conhecimento de todos os passos envolvidos no processo, excesso de tarefas da equipe de enfermagem, má formação profissional e insuficiência sobre o exame físico ${ }^{(16)}$.

No processo de implantação da $\mathrm{SAE}$, ressalta-se a importância de se avaliar os recursos humanos envolvidos, o interesse da instituição em aumentar o quantitativo e a capacitação dos profissionais. A educação continuada para enfermeiros assistenciais é apontada como mecanismo pelo qual pode se alcançar a mudança de comportamento(17). A assistência de enfermagem ao paciente em instituições hospitalares tem exigido da enfermagem uma atuação complexa, o que evoca a necessidade de capacitação científica e comprometimento ético, com vistas à melhoria do cuidado oferecido ${ }^{(18)}$.

\section{CONCLUSÕES}

O processo de enfermagem é o método por meio do qual a equipe de enfermagem presta a assistência de forma sistematizada, pautada em princípios científicos. Os benefícios gerados pela sua efetivação são reconhecidos não apenas pela literatura pertinente à temática, mas também pelos profissionais que estão diretamente vinculados à prática assistencial, conforme evidenciou o resultado do estudo. Percebeu-se ainda que há uma motivação destes em implantar a metodologia na prática, por acreditar em seus benefícios.

Entretanto, gerir mudanças com recursos materiais e humanos insuficientes constitui-se um desafio. Sugere-se o envolvimento da equipe gestora da organização, no intuito de prover os recursos necessários à implementação e à manutenção da $\mathrm{SAE}$, bem como investir na qualificação dos profissionais de enfermagem, de modo a favorecer o incremento da qualidade de assistência na perspectiva de revertê-la em benefícios à saúde dos pacientes, à própria equipe de enfermagem, demais profissionais da organização e à própria instituição prestadora de serviços de saúde.

\section{Referências}

1. Alfaro-LeFevre R. Aplicação do processo de enfermagem: uma ferramenta para o pensamento crítico. 7a ed. Porto Alegre: Artmed; 2010.

2. Horta W. Processo de enfermagem. Rio de Janeiro: Guanabara Koogan; 2011.

3. Brasil. Lei n. ${ }^{0} .498$, de 25 de junho de 1986. Dispōe sobre a Regulamentação do Exercício da Enfermagem e dá outras providências. Diário Oficial da República Federativa do Brasil, Brasilia (DF), 26 jun. 1986. Seção 1:1.

4. Conselho Federal de Enfermagem. Resolução n. 358, de 15 de outubro de 2009. Dispõe sobre a sistematização da assistência de enfermagem e a implementação do processo de enfermagem em ambientes, públicos ou privados, em que ocorre o cuidado profissional de enfermagem. Brasilia: COFEN; 2009.

5. Hermida PMV, Araújo IEM. Sistematização da assistência de enfermagem: subsídios para implantação. Rev Bras Enferm. 2006;59(5):675-9.

6. Gil AC. Como elaborar projetos de pesquisa. 4a ed. São Paulo: Atlas; 2009.

7. Ministério da Saúde (BR). Conselho Nacional de Saúde. Diretrizes e normas regulamentadoras de pesquisa envolvendo seres humanos. Resolução n. 196 , de 10 de outubro de 1996. Brasília: Ministério da Saúde; 1996.

8. Bardin L. Análise de conteúdo. São Paulo: Ediçōes 70; 2011.

9. Ralph SS, Taylor CM. Manual de diagnóstico de enfermagem. $7^{\mathrm{a}}$ ed. Rio de Janeiro: Guanabara Koogan; 2009.

10. Backes DS, Schwartz E. Implementação da sistematização da assistência de enfermagem: desafios e conquistas do ponto de vista gerencial. Ciênc Cuid Saúde. 2005;4(2):182-88.
11. Carvalho EC, Melo AS. O significado do processo de enfermagem para quem o ministra. Cogitare Enferm. 2008;13(3):352-60.

12. Barros ALBL, Lopes JL. A legalização e a sistematização da assistência de enfermagem. Enferm Foco. 2010;1(2):63-5.

13. Castilho NC, Ribeiro $\mathrm{PC}$, Chirelli MQ. A implementação da sistematizaçăo da assistência de enfermagem no serviço de saúde hospitalar do Brasil. Texto Contexto Enferm. 2009;18(2):180-9.

14. Tannure MC, Pinheiro AM. SAE-Sistematização da Assistência de Enfermagem: guia prático. 2a ed. Rio de Janeiro: Guanabara Koogan; 2010.

15. Sales LM, Afonso ESR, Santos TVC. Sistematizaçāo da Assistência de Enfermagem (SAE): uma pesquisa nas bases eletrônicas de dados. Rev Edu Meio Amb e Saúde. 2008;3(1):197-207.

16. Pokorski S, Moraes MA, Chiarelli R, Costanzi AP, Rabelo ER. Processo de enfermagem: da literatura à prática. $\mathrm{O}$ quê de fato nós estamos fazendo? Rev Latinoam Enferm. 2009;17(3):302-7.

17. Silva CMC, Azevedo SL, Valente GSC, Machado TF, Rosas AMMTF, Chrizóstimo MM. Diagnósticos de enfermería como instrumentos en la formación del enfermero: una revisión de la literatura. Enferm Glob. 2009;(17):1-12.

18. Vargas MAO, Luz AMH. Práticas seguras do/no cuidado de enfermagem no contexto hospitalar: é preciso pensar sobre isso e aquilo. Enferm Foco. 2010;1(1):23-7. 